\title{
Overexpression and clinical significance of MYC-associated zinc finger protein in pancreatic carcinoma
}

\author{
Xiaonian Zhul,* \\ Wei Luo ${ }^{2, *}$ \\ Wenjin Liang ${ }^{2}$ \\ Fen Tang ${ }^{3}$ \\ Chunhua Bei' \\ Yuan Ren' \\ Linyuan Qin' \\ Chao Tan' \\ Ying Zhang' \\ Shengkui Tan'
}

'School of Public Health, Guilin Medical University, Guilin, People's Republic of China; ${ }^{2}$ Department of Hepatobiliary Surgery, The Affiliated Hospital of Guilin Medical University, Guilin, People's Republic of China; ${ }^{3}$ Department of Hepatology, Nanxishan Hospital of Guangxi

Zhuang Autonomous Region, Guilin, People's Republic of China

*These authors contributed equally to this work

\author{
This article was published in the following Dove Press journal: \\ OncoTargets and Therapy \\ 12 December 2016 \\ Number of times this article has been viewed
}

Correspondence: Shengkui Tan School of Public Health, Guilin Medical University, 109 Huancheng North Road 2, Guilin 541004, Guangxi, People's Republic of China Tel/fax +8677322I 6868 Email sktan2008@sina.cn
Abstract: This study aimed to investigate the expression and clinical significance of MYC-associated zinc finger protein (MAZ) in pancreatic carcinoma (PC), and the biological functions of MAZ in PC cells. MAZ expression was detected in 57 PC tissues and 41 paired adjacent nontumor tissues by immunohistochemistry. Compared to the expression in adjacent nontumor tissues, MAZ was significantly higher expressed in PC tissues $(P<0.0001)$. In addition, MAZ expression had a significant correlation with certain clinical characteristics of $P C$ patients, such as age, tumor diameter, tumor number, and the serum level of CA199 $(P<0.05)$. The survival analysis showed that the survival time of PC patients with high expression of MAZ was significantly lower than patients with low expression of MAZ $(P=0.0365)$. After MAZ was knocked down in PANC-1 cells by RNA interference, the cells' ability to proliferate, invade, and migrate was decreased significantly $(P<0.01)$. Moreover, MAZ expression was found to be associated with Ki-67, a cell proliferation marker, in PC tissues, further supporting the idea that MAZ promotes PC cell proliferation. Our study clarifies an oncogenic role of MAZ in pathogenesis of $\mathrm{PC}$ and provides MAZ as a biomarker in the treatment and prognosis of PC.

Keywords: pancreatic carcinoma, MYC-associated zinc finger protein, prognosis, cell proliferation

\section{Introduction}

With incidence and mortality increasing year by year, cancer has become a major public health problem around the world. ${ }^{1-3}$ As one of the common malignant digestive tumors in China, the incidence of pancreatic carcinoma (PC) has increased quickly in recent years, and it is one among the top ten causes of cancer-related death. ${ }^{4}$ The mortality rate of PC ranks among the top four worldwide, ${ }^{5}$ this is the reason PC is called "the king of cancer". Moreover, the early diagnosis rate of PC patients is not high, and the majority of them are diagnosed at an advanced stage with very poor prognosis. Therefore, early diagnosis is crucial for PC patients. Current studies find that genetic and epigenetic regulations play an important role in pathogenesis of $\mathrm{PC}$, such as activation and mutation of $K R A S$ gene; ${ }^{6}$ deletion of tumor suppressor genes $p 16, T P 53$, SMAD4/DPC4, and BPCA2; and deregulation of microRNAs. ${ }^{7,8}$ However, there is currently no specific marker for the diagnosis of PC, although carbohydrate antigen 19-9 (CA199) is considered to be the gold standard for detection of PC. ${ }^{9}$ The increased CA199 is used for the diagnosis of PC by European Group on Tumor Markers and the US National Academy of Clinical Biochemistry, ${ }^{10}$ but increased levels of CA199 are also seen in nontumor patients, such as those with acute and chronic pancreatitis, 
hepatic cirrhosis, and obstructive jaundice. Therefore, new biomarkers are needed for early diagnosis, targeted therapy, and prognosis of PC besides CA199.

Zinc finger protein was found in a TFIIIA of Xenopus oocytes in early $1983 .{ }^{11,12}$ It is widely distributed in eukaryotic organisms and functions to regulate gene expression, cell differentiation, and embryo development. ${ }^{13}$ MYC-associated zinc finger protein (MAZ) is expressed in human heart, lung, brain, liver, skeletal muscle, prostate, and pancreas. Previous studies showed that MAZ protein not only activated transcription of some genes, such as $c-m y c, V E G F$, Ras gene family, and $P D P N,{ }^{14-18}$ but also terminated transcription of some genes, such as eNOS, Sp4, and p53. ${ }^{19,20}$ Recently, abnormal expression of MAZ was found in oblastoma, liposarcoma, breast cancer, and prostate cancer, and it was found to be closely related to the development of these tumors. ${ }^{16,21-23}$ However, the relationship between MAZ expression and clinicopathological characteristics and the prognosis of PC patients have not yet been reported.

In this study, the expression and clinical significance of MAZ in PC were investigated in paired PC and adjacent nontumor tissues by immunohistochemistry (IHC) and Western blot (WB), and the biological functions of MAZ were also explored in PC cells by RNA interference. Finally, we found that MAZ was overexpressed in PC tissues, and this was correlated with the prognosis of PC patients. In addition, MAZ was found to promote the proliferative, invasive, and migratory capacities of PC cells. This study suggests that $\mathrm{MAZ}$ is an oncogene in the pathogenesis of PC.

\section{Materials and methods}

\section{Patients and tissue samples}

Eight fresh $\mathrm{PC}$ tissues and paired adjacent nontumor tissues for Western blot were collected from PC patients undergoing surgery at the Affiliated Hospital of Guilin Medical University between 2015 and 2016. Another 57 PC tissues and 41 paired adjacent nontumor tissues for IHC analysis were collected from the Department of Pathology, the Affiliated Hospital of Guilin Medical University between 2007 and 2013. PC tissues were collected from PC patients diagnosed by clinical and pathological method, and the distance between PC tissues and adjacent nontumor tissues was greater than $2 \mathrm{~cm}$. All these PC patients underwent surgical treatment without prior radiotherapy or chemotherapy, and complete clinical data was available for all patients (Table 1). All specimens were obtained under agreements from the patients or their families along with written informed consent and approved by the ethics committee of the Affiliated Hospital of Guilin Medical University.
Table I Correlation between MAZ expression and clinicopathological characteristics of PC patients

\begin{tabular}{|c|c|c|c|c|c|}
\hline \multirow[t]{2}{*}{ Variables } & \multirow[t]{2}{*}{ Total } & \multicolumn{2}{|c|}{ MAZ staining } & \multirow[t]{2}{*}{$\chi^{2}$ value } & \multirow[t]{2}{*}{$P$-value } \\
\hline & & High & Low & & \\
\hline \multicolumn{6}{|l|}{ Sex } \\
\hline Male & 30 & 21 & 9 & 0.113 & 0.738 \\
\hline Female & 27 & 20 & 7 & & \\
\hline \multicolumn{6}{|l|}{ Age-yr } \\
\hline$\geq 50$ & 51 & 39 & 12 & 5.228 & 0.026 \\
\hline$<50$ & 6 & 2 & 4 & & \\
\hline \multicolumn{6}{|l|}{ Smoking } \\
\hline Yes & 19 & 13 & 6 & 0.168 & 0.683 \\
\hline No & 38 & 28 & 10 & & \\
\hline \multicolumn{6}{|c|}{ Alcohol intake } \\
\hline Yes & 26 & 18 & 8 & 0.167 & 0.684 \\
\hline No & 31 & 23 & 8 & & \\
\hline \multicolumn{6}{|c|}{ CAI 99 (U/mL) } \\
\hline$>37$ & 43 & 35 & 8 & 8.679 & 0.005 \\
\hline$\leq 37$ & 14 & 6 & 8 & & \\
\hline \multicolumn{6}{|c|}{ Tumor diameter $(\mathrm{cm})$} \\
\hline$\leq 5$ & 26 & 15 & 11 & 5.057 & 0.029 \\
\hline$>5$ & 31 & 26 & 5 & & \\
\hline \multicolumn{6}{|c|}{ Tumor grade } \\
\hline I & 11 & 9 & 2 & 0.644 & 0.426 \\
\hline II + III & 46 & 32 & 14 & & \\
\hline \multicolumn{6}{|c|}{ Tumor number } \\
\hline 1 & 49 & 39 & 10 & 11.917 & 0.001 \\
\hline$\geq 2$ & 8 & 2 & 6 & & \\
\hline \multicolumn{6}{|l|}{ Metastasis } \\
\hline Yes & 42 & 28 & 14 & 2.197 & 0.144 \\
\hline No & 15 & 13 & 2 & & \\
\hline
\end{tabular}

Notes: Bold values indicate significance. $P$-value is based on the $\chi^{2}$ test. Abbreviations: Age-yr, Age-year; MAZ, MYC-associated zinc finger protein; $\mathrm{PC}$, pancreatic carcinoma.

\section{Immunohistochemistry assay and scoring}

Paraffin-embedded tissues were first heated at $60^{\circ} \mathrm{C}$ for $1 \mathrm{~h}$ and then dewaxed using dimethylbenzene. After hydration in a gradient series of ethanol, these tissues were placed in EDTA buffer $(\mathrm{pH}=8.0)$ for high-pressure heating repair and then soaked in 3\% hydrogen peroxide solution for $15 \mathrm{~min}$ to remove endogenous peroxidase. The sample was then incubated with primary antibody MAZ (Abcam, Cambridge, MA, USA; at a dilution of $1: 200$ ) at $37^{\circ} \mathrm{C}$ for $1 \mathrm{~h}$ after blocking with horse serum at room temperature for $30 \mathrm{~min}$. Then, second antibody (Maixin company, Fuzhou, People's Republic of China) was added and incubated at $37^{\circ} \mathrm{C}$ for 30 min. Then, the tissues were treated with DAB for 3 5 min until the appropriate color was observed under a microscope. Finally, they were counterstained with hematoxylin, rinsed with tap water, dehydrated, made transparent, and fixed.

Five fields were randomly selected for scoring the IHC results. Scoring was done using a microscope at high magnification. The percentage of cells that stained positive, and 
staining intensity, were counted for evaluation. 1) Staining intensity score: colorless was 0 points, light yellow was 1 point, yellow-brown was 2 points, and brown was 3 points. 2) Scoring for the percentage of positive cells: negative was 0 points, less than $10 \%$ was 1 point, $11 \%-50 \%$ was 2 points, $51 \%-75 \%$ was 3 points, and more than $75 \%$ was 4 points. The final score was the product of the abovementioned two scores. Scores $>6$ were defined as high expression and $\leq 6$ as low expression.

\section{Cell culture}

The human pancreatic cell line HPDE6C7 and other pancreatic cancer cell lines were purchased from ATCC cell bank. HPDE6C7 and PANC-1 cells were cultured in Dulbecco's Modified Eagle's Medium containing 10\% fetal bovine serum (FBS). ASPC-1 and BXPC-3 cells were cultured in Roswell Park Memorial Institute-1640 medium containing 10\% FBS, while CFPAC-1 cells were cultured in Iscove's Modified Dulbecco's Medium containing 10\% FBS. All cells were incubated at $37^{\circ} \mathrm{C}$ with $5 \% \mathrm{CO}_{2}$.

\section{Plasmid construction}

Four shRNAs targeting MAZ (shMAZ) and a negative control shGFP were constructed by Genechem (Shanghai, People's Republic of China). The target sequences were as follows: shMAZ-1\#, GCTTATATTTCGGACCACA; shMAZ-2\#, GCCCTTCAAATGTGAGAAA, shMAZ-3\#; GTTCAAGAACGGCTACAAT; and shMAZ-4\#, GGCCAT GTTCCCGGTGTTT. These shRNAs were transferred to pancreatic cancer cell lines and their knockdown effect was validated by WB. Finally, shMAZ-1\# and shMAZ-2\# were chosen for subsequent experiments.

\section{Western blot}

Cells or tissue samples were lysed with RIPA buffer containing $1 \%$ PMSF, and then the concentration of protein was determined by BCA. Twenty micrograms of protein per well was loaded for SDS-PAGE. After electrophoresis, the proteins were transferred to PVDF membranes and coated with specific primary antibodies overnight at $4^{\circ} \mathrm{C}$ after blocking with $5 \%$ fat-free milk. Then, the membranes were washed with TBST buffer and incubated with secondary antibodies at room temperature for $1 \mathrm{~h}$. Finally, the bands were visualized by chemiluminescence.

\section{Cell proliferation}

Cell proliferation was assessed at $6 \mathrm{~h}, 12 \mathrm{~h}, 24 \mathrm{~h}, 48 \mathrm{~h}$ and $72 \mathrm{~h}$ after cell seeding using the CCK8 kit according to the manufacturer's protocol. Cells were grown in triplicate in a 96-well plate at a density of 8,000 cells/well. CCK-8 reagent was added at the setting time $(6 \mathrm{~h}, 12 \mathrm{~h}, 24 \mathrm{~h}, 48 \mathrm{~h}$ and $72 \mathrm{~h}$ after cell seeding) and incubated for $1 \mathrm{~h}$ at $37^{\circ} \mathrm{C}$. Then, these cells were used to detect optical density values at $450 \mathrm{~nm}$ on a microplate reader.

\section{Colony formation assay}

Cells were grown in triplicate in a 6-well plate at a density of 500 cells/well. After culturing for 2 weeks, cells were washed with phosphate-buffered saline (PBS) two times. Then, the cells were fixed with $4 \%$ paraformaldehyde for $15 \mathrm{~min}$ and stained using crystal violet for $2 \mathrm{~h}$. Finally, they were washed with deionized water three times and then photographed. The cell colonies were counted under a microscope.

\section{Cell apoptosis detection}

Cell apoptosis was determined using Annexin V-FITC Apoptosis Detection Kit (Beyotime, Jiangsu, People's Republic of China) according to the supplier's instructions. Each cell line was tested at least three times and apoptotic cells determined quantitatively by flow cytometry.

\section{Cell invasion and migration analysis}

Cell invasion was detected using Matrigel (BD, Franklin lakes, NJ, USA)-coated BD Transwell chambers, and cell migration was detected using BD Transwell chamber without Matrigel coating. The chamber aperture was $8 \mu \mathrm{m}$. The chamber was put in a 24-well plate with serum-free medium and incubated for $1 \mathrm{~h}$ to enable activation. Then, $2 \times 10^{4}$ cells in $100 \mu \mathrm{L}$ serum-free medium was added to the upper chamber and $600 \mu \mathrm{L}$ medium with $10 \%$ FBS was added to the lower chamber. After $24 \mathrm{~h}$ of incubation, cells were fixed with $4 \%$ paraformaldehyde and stained with $1 \%$ crystal violet. The Matrigel and the cells that remained were wiped off. After washing with PBS, the cells were photographed and counted under a microscope.

\section{Statistical analysis}

All the results were analyzed using SPSS 19.0 (IBM, Armonk, NY, USA). The correlation between clinicopathological parameters and MAZ expression was analyzed using $\chi^{2}$ test. The survival probability was estimated by Kaplan-Meier method, and the comparison of survival curves between groups was done with the log rank test. The level of statistical significance was set at $P<0.05$ for all tests.

\section{Results \\ MAZ is highly expressed in PC tissues and associated with prognosis of PC patients}

To assess the relationship between MAZ and PC, the expression of MAZ protein was first detected in eight paired fresh 
PC, and adjacent nontumor tissues, by WB. As shown in Figure 1A, increased expression of MAZ protein was found in 7 of $8(87.5 \%)$ PC tissues, suggesting MAZ was overexpressed in PC tissues. Then we conducted IHC analysis to determine the expression of MAZ in another 57 PC tissues. As shown in Figure 1B, MAZ was expressed both in cytoplasm and nucleus, but mainly located in the cytoplasm. Compared to the adjacent nontumor tissues, MAZ was significantly higher in PC tissues $(P<0.0001$, Figure $1 C)$, which is consistent with the WB result.

To identify the prognostic value of MAZ for PC, clinicopathological characteristics of the 57 PC patients were collected to analyze the correlation between the expression of MAZ and PC (Table 1). The patients were divided in two groups according to the MAZ staining score by IHC. After comparing with $\chi^{2}$ test, there was a significant difference between the high MAZ expression group and the low MAZ expression group in age, tumor diameter, tumor number, and CA199 level $(P<0.05)$, while MAZ expression had no significant relationship with other characteristics such as sex, smoking, alcohol intake, tumor grade, and metastasis $(P>0.05)$. The correlation between MAZ expression and the prognosis of PC patients was also analyzed by Kaplan-Meier method. As shown in Figure 1D, there were 41 PC patients
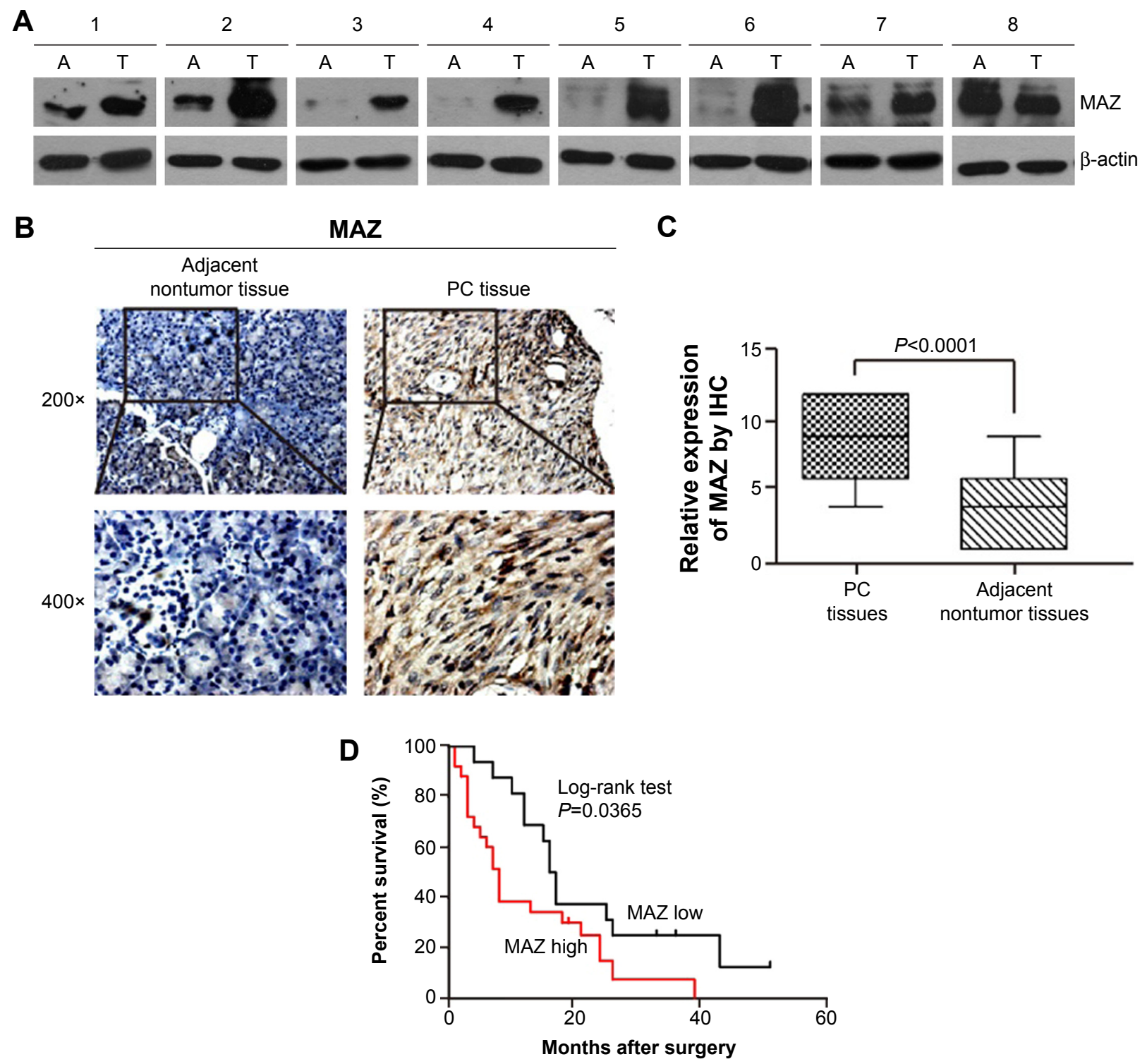

Figure I MAZ is highly expressed in PC and correlated with prognosis of $\mathrm{PC}$ patients.

Notes: (A) MAZ protein expression was detected by WB in PC and adjacent nontumor tissues. (B) MAZ protein expression was detected in representative PC and adjacent nontumor tissues by IHC analysis at $200 \times$ and $400 \times$ magnification. (C) Statistical analysis of MAZ expression was performed in 57 PC and 46 adjacent nontumor tissues. $P$-value is based on the $\chi^{2}$ test. (D) Correlation of MAZ expression with survival time of PC patients was conducted by Kaplan-Meier survival analysis.

Abbreviations: A, adjacent nontumor tissues; IHC, immunohistochemistry; MAZ, MYC-associated zinc finger protein; PC, pancreatic carcinoma; T, PC tissues; WB, Western blot. 
with high expression of MAZ and 16 with low expression of MAZ. Compared with the low MAZ expression group, the survival time of the high MAZ expression group was significantly decreased after surgery (log-rank test, $P=0.0365$ ). These results indicate that overexpressed MAZ is associated with poor prognosis of PC patients, suggesting that MAZ might play a role in $\mathrm{PC}$ pathogenesis.

\section{MAZ is overexpressed in PC cells}

To explore the role MAZ plays in PC pathogenesis, we detected the expression of MAZ protein in four pancreatic cancer cell lines ASPC-1, CFPAC-1, PANC-1, and BXPC-3 by WB. As shown in Figure 2A, compared with the normal pancreatic cell line HPDE6C7, MAZ had a higher expression in these four PC cell lines, especially in PANC-1 cells. Therefore, PANC-1 cells were selected for subsequent experiments. At the same time, we purchased four shMAZ to knock down MAZ expression and a negative control shGFP. After transferring these shRNAs to PANC-1 cells, the four shMAZ had a significant knockdown effect on MAZ expression compared to shGFP (Figure 2B). As both shMAZ-1\# and shMAZ-2\# knocked down MAZ expression more than $70 \%$, they were chosen to construct MAZ knockdown cell lines (named as PANC-1-shMAZ-1\# and PANC-1-shMAZ-2\#) to explore the roles of MAZ in PC pathogenesis.
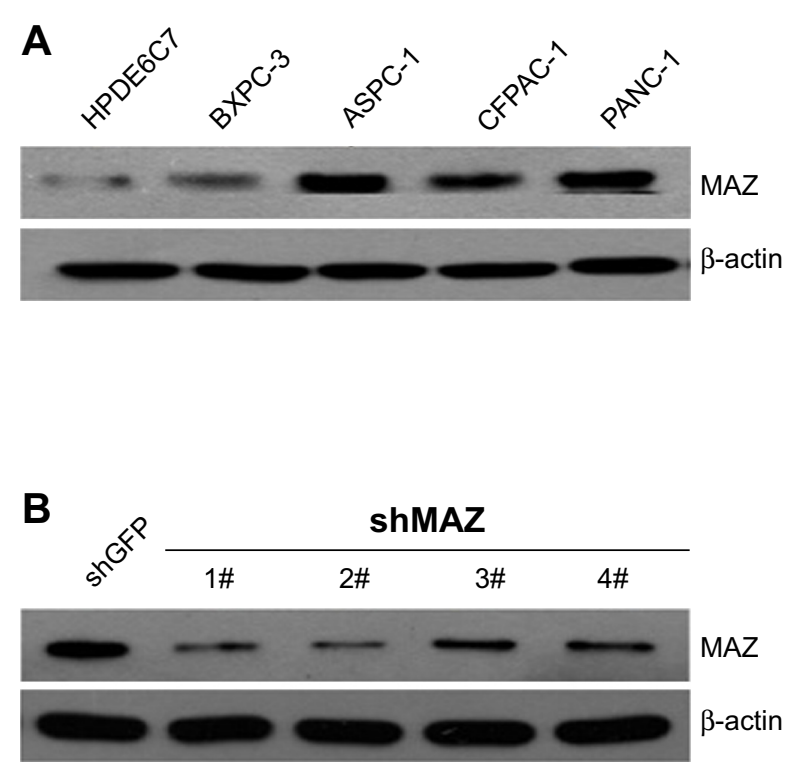

Figure $2 M A Z$ is overexpressed in PC cells.

Notes: (A) MAZ protein expression in normal pancreatic cell line HPDE6C7 and four PC cell lines as indicated was detected by WB (right panel, gray scan results). (B) MAZ protein expression in PANC-I cells introduced with four specific shRNAs targeting MAZ and a control shGFP (right panel, gray scan results).

Abbreviations: MAZ, MYC-associated zinc finger protein; PC, pancreatic carcinoma; WB, Western blot.

\section{MAZ promotes the proliferation of PC cells}

To assess the effects of MAZ knockdown on cell proliferation, we performed CCK-8 and colony formation assay in the constructed PANC-1-shMAZ cells. Compared to PANC1-shGFP cells, MAZ expression in the PANC-1-shMAZ-1\# and PANC-1-shMAZ-2\# cells was decreased by $70 \%$ and $80 \%$, respectively (Figure 3A). Moreover, the proliferation of both PANC-1-shMAZ-1\# and PANC-1-shMAZ-2\# cells was decreased significantly (Figure 3B, $P<0.01$ ). In accordance with this result, colony number of PANC-1-shMAZ cells was also less than that of PANC-1-shGFP cells (Figure 3C). In addition, the apoptosis in PANC-1-shMAZ cells was more than that of PANC-1-shGFP cells (Figure 3D). These results reveal that MAZ can promote the proliferation and inhibit the apoptosis of PC cells.

\section{MAZ promotes metastasis of $P C$ cells}

As MAZ expression was correlated with the prognosis of $\mathrm{PC}$ patients, we tried to find out whether MAZ influenced the metastasis of PC cells. We detected the cell ability of invasion and migration in PANC-1 cells through Transwell assay. As shown in Figure 4A and 4B, we found that the downregulation of MAZ in PANC-1 cells significantly decreased the invasion and migration ability compared with the control PANC-1-shGFP cells $(P<0.01)$. This result shows that MAZ
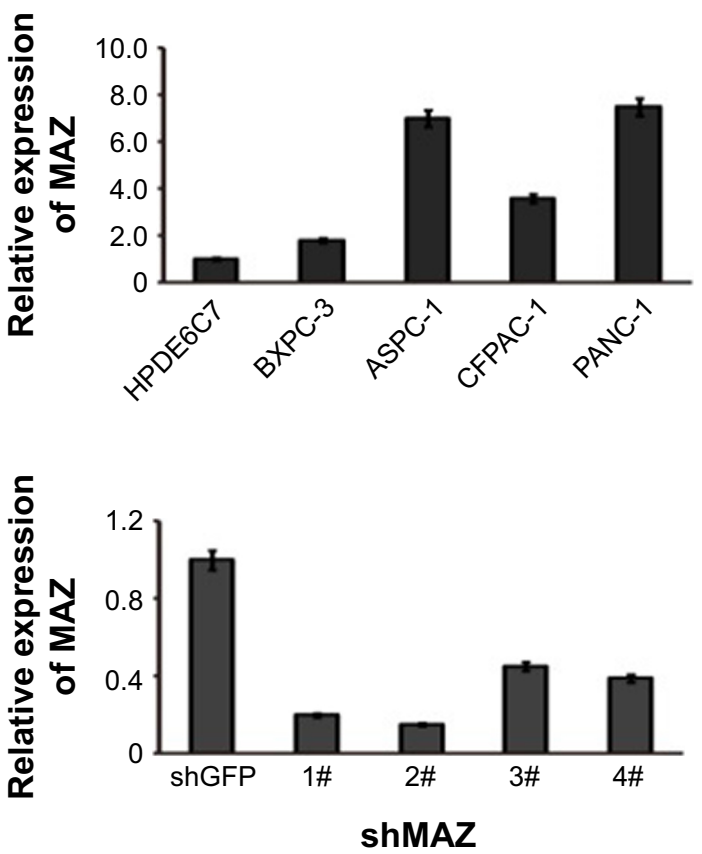
A

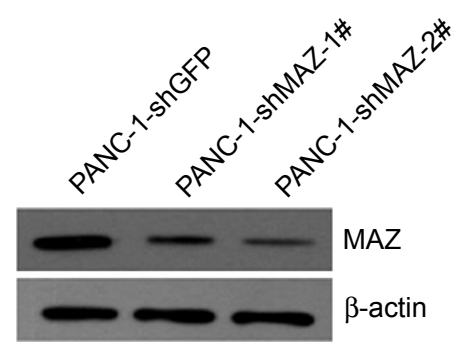

C

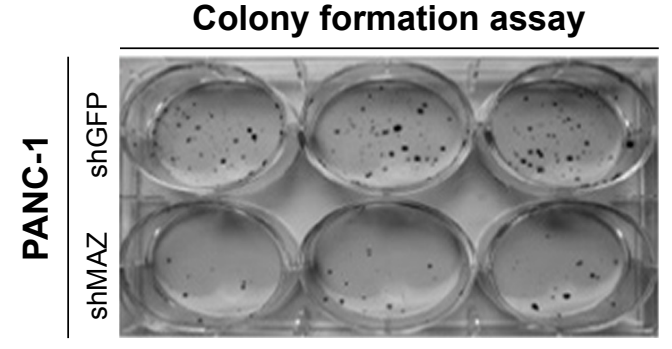

B
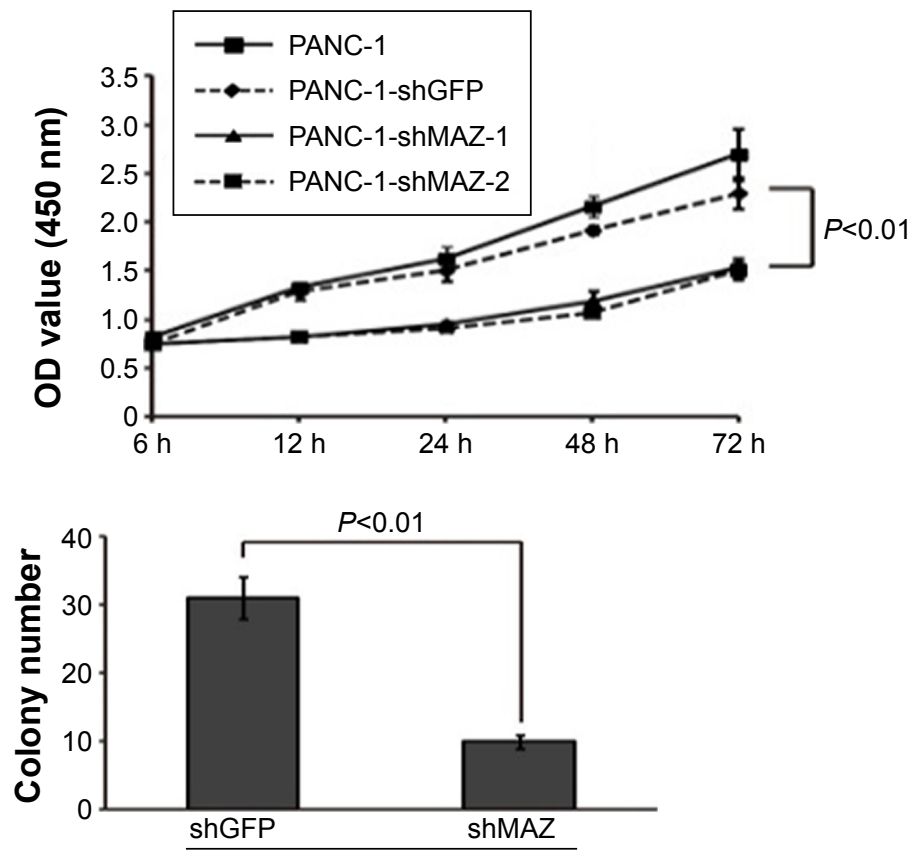

PANC-1

D

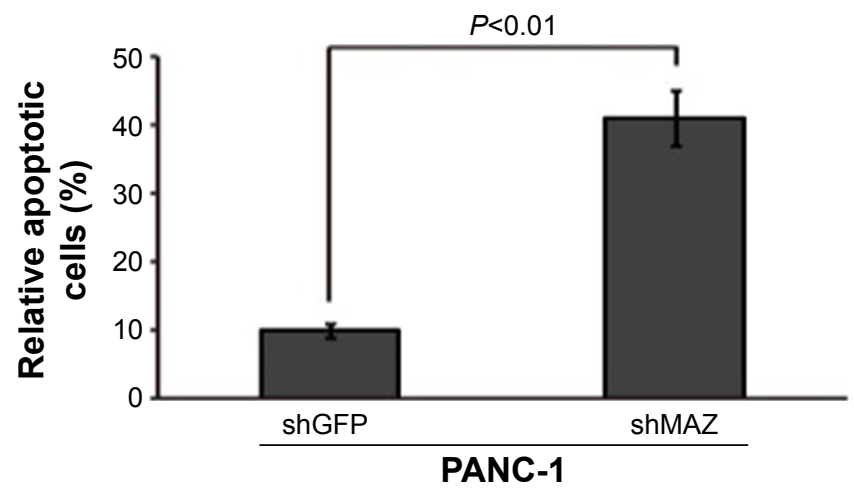

Figure 3 MAZ promotes the proliferation of PC cells.

Notes: (A) MAZ protein expression was detected by WB in constructed PANC-I-shMAZ-I\# and PANC-I-shMAZ-2\# cells. (B) and (C) Cell proliferation was detected by CCK-8 and colony formation assay. (D) Distribution of annexin $V$ binding was determined by flow cytometry. Data are represented as the mean \pm standard errors of three independent experiments. $P$-value is based on the Student $t$-test compared to PANC-I-shGFP cells.

Abbreviations: MAZ, MYC-associated zinc finger protein; PC, pancreatic carcinoma; WB, Western blot.

also has an effect on the invasion and migration of PC cells, which might result in a poor prognosis of $\mathrm{PC}$ patients.

\section{The correlation between MAZ expression and $\mathrm{Ki}-67$}

To further clarify the role MAZ plays in PC cell proliferation, we detected the expression of Ki-67, a marker for cell proliferation, in PC and adjacent nontumor tissues in which MAZ had been studied. As shown in Figure 5A, Ki-67 was expressed in both PC and adjacent nontumor tissues. However, there was a significantly higher expression of Ki-67 in $\mathrm{PC}$ tissues than in adjacent nontumor tissues $(P<0.05$, Figure 5B). In addition, we found that the expression of $\mathrm{Ki}-67$ was associated with tumor diameter. Ki-67 expression in PC tissues with tumor diameter more than $5 \mathrm{~cm}$ was significantly higher than that in PC tissues with tumor diameter less than $5 \mathrm{~cm}(P<0.05$, Figure 5C and 5D). As MAZ and Ki-67 were both overexpressed in PC tissues, we conducted a correlation analysis between them. As shown in Table 2, there was a positive correlation between the expression levels of MAZ and Ki-67 in PC tissues ( $r=0.635$, $P=0.000$ ), suggesting that $\mathrm{MAZ}$ is involved in pathogenesis of $\mathrm{PC}$ through promoting PC cell proliferation.

\section{Discussion}

PC is one kind of malignant solid tumors causing the highest mortality and is a serious health problem in the world. ${ }^{24}$ Because of its occult onset, most PC patients are diagnosed at a late stage, resulting in only about $10 \%-20 \%$ of PC patients eligible for radical surgery. The average survival time of 
A

PANC-1

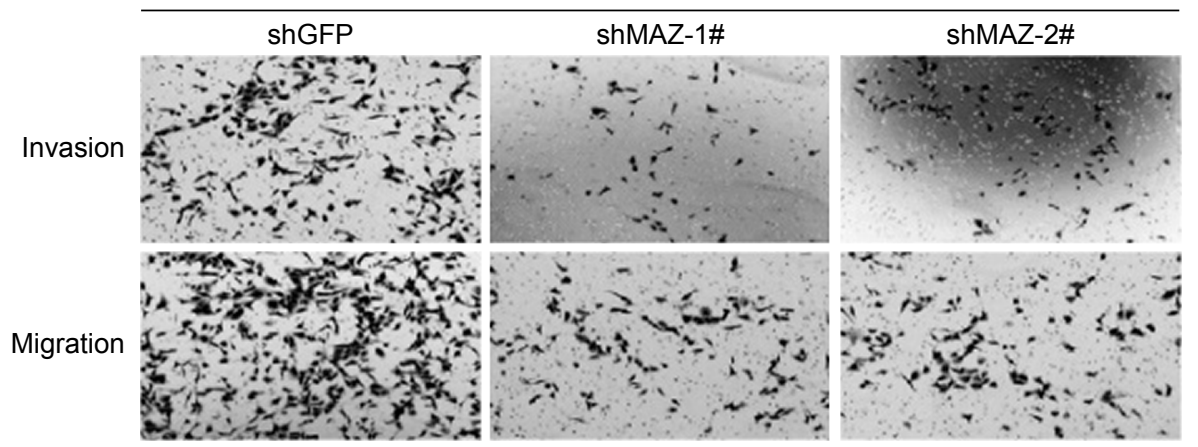

B

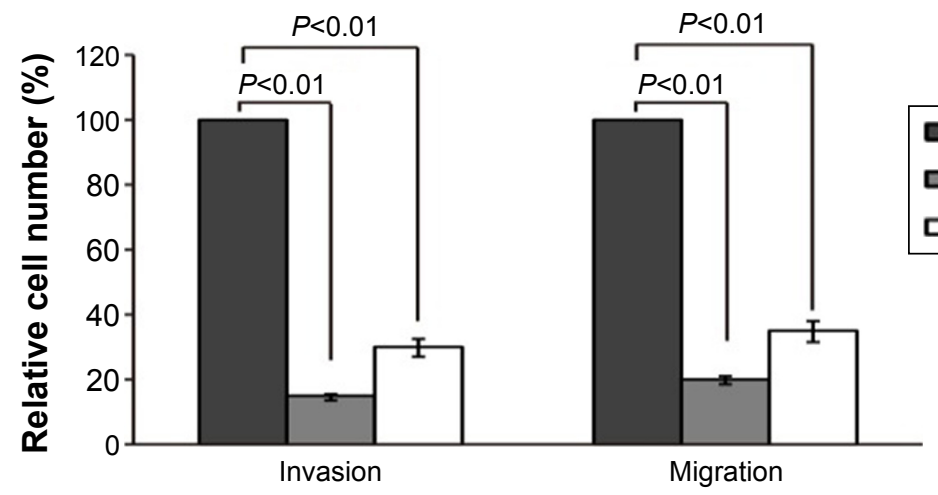

口 PANC-1-shGFP

口 PANC-1-shMAZ-1

口 PANC-1-shMAZ-2

Figure $4 \mathrm{MAZ}$ promotes invasion and migration of PC cells.

Notes: (A) and (B): Invasion and migration ability of cells was analyzed by Transwell assay. P-value is based on the Student $t$-test compared to PANC-I-shMAZ-I\# and panc-I-shMAZ-2\# cells. All results are from three independent experiments.

Abbreviations: MAZ, MYC-associated zinc finger protein; PC, pancreatic carcinoma.

A

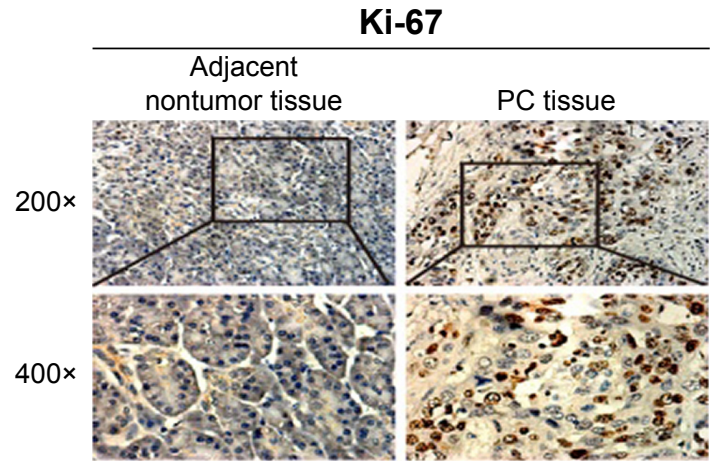

C

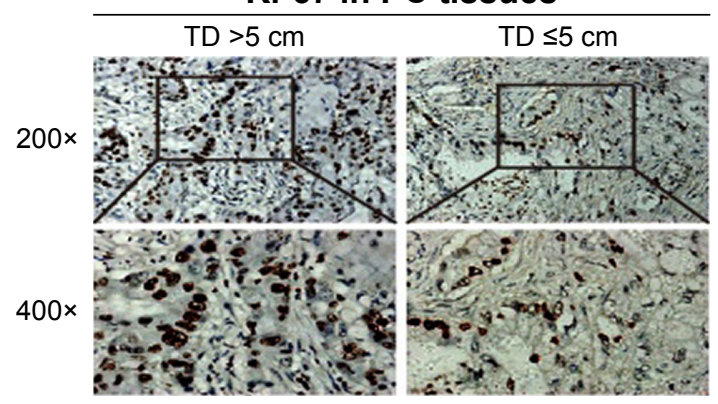

B

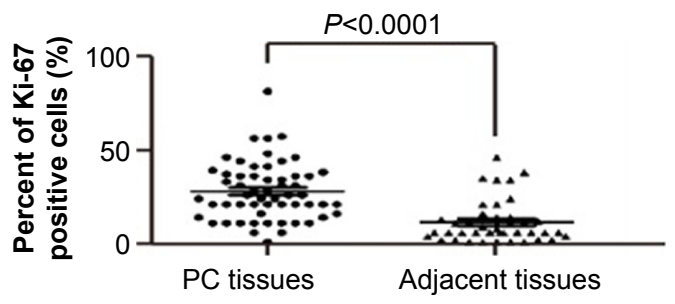

D

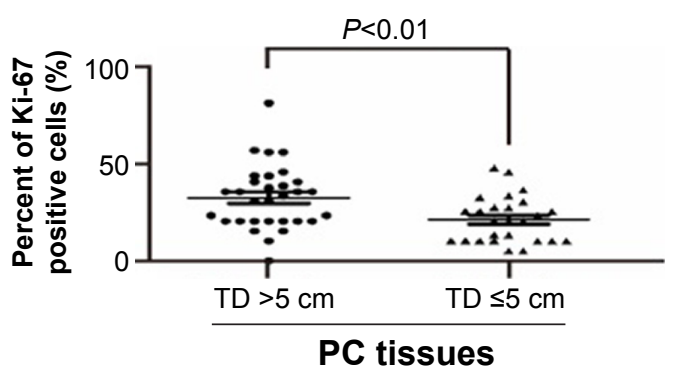

Figure 5 Ki-67 expression in PC and adjacent nontumor tissues.

Notes: (A) Ki-67 expression was detected in representative PC and adjacent nontumor tissues by IHC analysis at 200× and 400× magnification. (B) Statistical analysis of $\mathrm{Ki}-67$ expression was performed in 57 PC and 46 adjacent nontumor tissues. $P$-value is based on the $\chi^{2}$ test. (C) Ki-67 expression was detected in representative PC tissues with TD $>5 \mathrm{~cm}$ or $\leq 5 \mathrm{~cm}$ by IHC analysis at $200 \times$ and $400 \times$ magnification. (D) Statistical analysis of Ki-67 expression was performed in 3 I PC tissues with TD $>5 \mathrm{~cm}$ and 26 PC tissues with TD $\leq 5 \mathrm{~cm}$. $P$-value is based on the $\chi^{2}$ test.

Abbreviations: PC, pancreatic carcinoma; IHC, immunohistochemistry; TD, tumor diameter. 
Table 2 Relationship between MAZ and Ki-67 in PC tissues

\begin{tabular}{lllllll}
\hline MAZ & $\mathbf{n}$ & Ki-67 & & $\chi^{2}$ & $\boldsymbol{r}$ & P-value \\
\cline { 3 - 4 } & & High & Low & & & \\
\hline High & 41 & 39 & 2 & 22.991 & 0.635 & $\mathbf{0 . 0 0 0}$ \\
Low & 16 & 6 & 10 & & & \\
\hline
\end{tabular}

Note: Bold values indicate significance.

Abbreviations: MAZ, MYC-associated zinc finger protein; PC, pancreatic carcinoma.

PC patients is only about 6 months. ${ }^{25}$ Tumorigenesis is a multistep process in which multiple genes are involved, such as abnormal activation of oncogenes and inactivation of tumor suppressor genes, causing changes in a series of downstream signaling cascades. ${ }^{26}$ Zinc finger protein is implicated in expression regulation of many genes, cell differentiation, and tumorigenesis. ${ }^{13}$ As a member of zinc finger protein, MAZ not only plays an important role in the regulation of colitis caused by oxygen lack or blood-tumor barrier, ${ }^{27,28}$ but also in the regulation of $c-m y c, V E G F, p 53, R a s$, and the caveolae structure protein caveolin-1. ${ }^{15-17,27,29}$ Previous studies showed that MAZ was overexpressed in glioblastoma, breast cancer, prostate cancer, and liposarcoma, indicating a poor prognosis of patients. ${ }^{16,17,22,30,31}$ However, the relationship between MAZ and $\mathrm{PC}$ has not yet been reported, and the role MAZ plays in PC is not yet fully clear.

Our study shows high expression of MAZ in PC tissues for the first time. In addition, we find out that high expression of MAZ is correlated with age, tumor diameter, tumor number, and serum CA199 level of PC patients. Furthermore, the survival analysis shows that the prognosis of PC patients with high expression of MAZ is poorer than those with low expression of MAZ, which is in accordance with the previous report which showed that upregulated MAZ in breast cancer affects the prognosis of breast cancer patients. ${ }^{30}$

MAZ was found to be upregulated in prostate cancer cells and positively regulated transcription of androgen receptor. When MAZ was silenced, cell proliferation and the ability to invade and migrate were decreased in prostate cancer cells. ${ }^{22} \mathrm{MAZ}$ could also promote tumor angiogenesis through transcriptional regulation of VEGF in human glioblastoma. ${ }^{17}$ In addition, MAZ was reported to regulate cell proliferation and apoptosis of liposarcoma cells by directly regulating GNDF, an effector in RET signaling pathway, cooperating with SPN1. ${ }^{21}$ These studies indicate that MAZ functions in tumor cell proliferation, invasion, and migration. Our study also confirmed these functions of MAZ through knocking down MAZ in PANC-1 cells, suggesting that MAZ plays an oncogenic role in pathogenesis of PC. The correlation between MAZ and cell proliferation marker Ki-67 further supports this conclusion. ${ }^{32}$ However, the specific regulating mechanism between MAZ and Ki-67 needs further exploration. Although we found MAZ promoted invasion and migration of PC cells in vitro, there was no significant correlation between MAZ and metastasis of PC patients $(P>0.05)$. This may be due to the small sample size of our study, and we will increase the number of PC tissues to investigate this in the future.

In summary, we found that MAZ was overexpressed in PC tissues and associated with poor prognosis of PC patients. Moreover, MAZ promoted the proliferation, invasion, and migration of human PC cells, suggesting an oncogenic role of MAZ in pathogenesis of PC. These results prove that MAZ can be used as a biomarker in the treatment and prognosis of PC.

\section{Acknowledgment}

This work was supported by the National Nature Science Foundation of China (NSFC, 81460515), and Scientific Research Project of Guangxi Universities and Colleges (KY2015ZD088).

\section{Disclosure}

The authors report no conflicts of interest in this work.

\section{References}

1. Peery AF, Crockett SD, Barritt AS, et al. Burden of gastrointestinal, liver, and pancreatic diseases in the United States. Gastroenterology. 2015;149(7):1731-1741.e3.

2. Quaresma M, Coleman MP, Rachet B. 40-year trends in an index of survival for all cancers combined and survival adjusted for age and sex for each cancer in England and Wales, 1971-2011: a population-based study. Lancet. 2015;385(9974):1206-1218.

3. Zheng R, Zeng H, Zhang S, Chen T, Chen W. National estimates of cancer prevalence in China, 2011. Cancer Lett. 2016;370(1):33-38.

4. Chen W. Cancer statistics: updated cancer burden in China. Chin J Cancer Res. 2015;27(1):1.

5. Siegel RL, Miller KD, Jemal A. Cancer statistics, 2016. CA Cancer J Clin. 2016;66(1):7-30.

6. Farina Sarasqueta A, Moerland E, de Bruyne H, et al. SNaPshot and StripAssay as valuable alternatives to direct sequencing for KRAS mutation detection in colon cancer routine diagnostics. J Mol Diagn. 2011;13(2):199-205.

7. Han S, Gonzalo DH, Feely M, et al. The pancreatic tumor microenvironment drives changes in miRNA expression that promote cytokine production and inhibit migration by the tumor associated stroma. Oncotarget. Epub 2016 Jul 20.

8. Iacobuzio-Donahue CA, Fu B, Yachida S, et al. DPC4 gene status of the primary carcinoma correlates with patterns of failure in patients with pancreatic cancer. J Clin Oncol. 2009;27(11):1806-1813.

9. Poruk KE, Gay DZ, Brown K, et al. The clinical utility of CA 19-9 in pancreatic adenocarcinoma: diagnostic and prognostic updates. Curr Mol Med. 2013;13(3):340-351.

10. Duffy MJ, Sturgeon C, Lamerz R, et al. Tumor markers in pancreatic cancer: a European Group on Tumor Markers (EGTM) status report. Ann Oncol. 2010;21(3):441-447. 
11. Lee MS, Gippert GP, Soman KV, Case DA, Wright PE. Three-dimensional solution structure of a single zinc finger DNA-binding domain. Science. 1989;245(4918):635-637.

12. Miller J, McLachlan AD, Klug A. Repetitive zinc-binding domains in the protein transcription factor IIIA from Xenopus oocytes. EMBOJ. 1985;4(6):1609-1614.

13. Hoovers JM, Mannens M, John R, et al. High-resolution localization of 69 potential human zinc finger protein genes: a number are clustered. Genomics. 1992;12(2):254-263.

14. Cogoi S, Zorzet S, Rapozzi V, Geci I, Pedersen EB, Xodo LE. MAZ-binding G4-decoy with locked nucleic acid and twisted intercalating nucleic acid modifications suppresses KRAS in pancreatic cancer cells and delays tumor growth in mice. Nucleic Acids Res. 2013;41(7):4049-4064.

15. Izzo MW, Strachan GD, Stubbs MC, Hall DJ. Transcriptional repression from the c-myc P2 promoter by the zinc finger protein ZF87/MAZ. J Biol Chem. 1999;274(27):19498-19506.

16. Ray A, Ray BK. Induction of Ras by SAF-1/MAZ through a feedforward loop promotes angiogenesis in breast cancer. Cancer Med. 2015;4(2):224-234.

17. Smits $M$, Wurdinger $T$, van het Hof B, et al. Myc-associated zinc finger protein (MAZ) is regulated by miR-125b and mediates VEGF-induced angiogenesis in glioblastoma. FASEB J. 2012;26(6):2639-2647.

18. Yao Y, Ma J, Xue Y, et al. MiR-449a exerts tumor-suppressive functions in human glioblastoma by targeting Myc-associated zinc-finger protein. Mol Oncol. 2015;9(3):640-656.

19. Song J, Mangold M, Suske G, et al. Characterization and promoter analysis of the mouse gene for transcription factor Sp4. Gene. 2001 264(1):19-27.

20. Teichert AM, Karantzoulis-Fegaras F, Wang Y, et al. Characterization of the murine endothelial nitric oxide synthase promoter. Biochim Biophys Acta. 1998;1443(3):352-357.

21. Franz H, Greschik H, Willmann D, et al. The histone code reader SPIN1 controls RET signaling in liposarcoma. Oncotarget. 2015; 6(7):4773-4789.
22. Jiao L, Li Y, Shen D, et al. The prostate cancer-up-regulated Mycassociated zinc-finger protein (MAZ) modulates proliferation and metastasis through reciprocal regulation of androgen receptor. Med Oncol. 2013;30(2):570.

23. Wang X, Southard RC, Allred CD, Talbert DR, Wilson ME, Kilgore MW. MAZ drives tumor-specific expression of PPAR gamma 1 in breast cancer cells. Breast Cancer Res Treat. 2008;111(1):103-111.

24. Warshaw AL, Fernandez-del Castillo C. Pancreatic carcinoma. N Engl J Med. 1992;326(7):455-465.

25. Bornman PC, Beckingham IJ. ABC of diseases of liver, pancreas, and biliary system. Pancreatic tumours. Bmj. 2001;322(7288):721-723.

26. Lin H, van den Esschert J, Liu C, van Gulik TM. Systematic review of hepatocellular adenoma in China and other regions. J Gastroenterol Hepatol. 2011;26(1):28-35.

27. Xie L, Xue X, Taylor M, et al. Hypoxia-inducible factor/MAZ-dependent induction of caveolin-1 regulates colon permeability through suppression of occludin, leading to hypoxia-induced inflammation. Mol Cell Biol. 2014;34(16):3013-3023.

28. Zhao L, Wang P, Liu Y, Ma J, Xue Y. miR-34c regulates the permeability of blood-tumor barrier via MAZ-mediated expression changes of ZO-1, occludin, and claudin-5. J Cell Physiol. 2015;230(3): 716-731.

29. Lee WP, Lan KH, Li CP, Chao Y, Lin HC, Lee SD. Akt phosphorylates myc-associated zinc finger protein (MAZ), releases P-MAZ from the p53 promoter, and activates p53 transcription. Cancer Lett. 2016;375(1):9-19.

30. Peurala H, Greco D, Heikkinen T, et al. MiR-34a expression has an effect for lower risk of metastasis and associates with expression patterns predicting clinical outcome in breast cancer. PloS One. 2011;6(11):e26122.

31. Zaytseva YY, Wang X, Southard RC, Wallis NK, Kilgore MW. Down-regulation of PPARgamma1 suppresses cell growth and induces apoptosis in MCF-7 breast cancer cells. Mol Cancer. 2008;7:90.

32. Cuylen S, BlaukopfC, Politi AZ, et al. Ki-67 acts as a biological surfactant to disperse mitotic chromosomes. Nature. 2016;535(7611):308-312.
OncoTargets and Therapy

\section{Publish your work in this journal}

OncoTargets and Therapy is an international, peer-reviewed, open access journal focusing on the pathological basis of all cancers, potential targets for therapy and treatment protocols employed to improve the management of cancer patients. The journal also focuses on the impact of management programs and new therapeutic agents and protocols on

\section{Dovepress}

patient perspectives such as quality of life, adherence and satisfaction. The manuscript management system is completely online and includes a very quick and fair peer-review system, which is all easy to use. Visit http://www.dovepress.com/testimonials.php to read real quotes from published authors. 